\title{
Author Correction: SARS-CoV-2 and HIV-1 - A Tale of Two Vaccines
}

\section{Barton F. Haynes (D)}

Correction to: Nature Reviews Immunology https://www.nature.com/articles/s41577-021-00589-w, published online 16 July 2020.

The article contained an error in the grant number listed in the acknowledgement. The corrected version reads: "B.F.H. is supported by the NIH, NIAID, Division of AIDS NIAID grant UM1 Al144371 Consortium for HIV/AIDS Vaccine Development".

https://doi.org/10.1038/s41577-021-00671-3 I Published online 13 December 2021

(๑) Springer Nature Limited 2021 